\title{
Dinámicas asociativas del tejido empresarial en Cartagena de Indias (1860-1960)
}

\author{
Abel Del Río Cortina \\ Universidad EAN \\ Colombia \\ Jorge Elías Caro \\ Colombia
}

Universidad de Magdalena 


\title{
Dinámicas asociativas del tejido empresarial en Cartagena de Indias (1860-1960) ${ }^{1}$
}

\author{
Abel Del Río Cortina ${ }^{2}$ iD , Jorge Elías Caro ${ }^{3}$ (D) \\ 2 Universidad EAN-Colombia \\ 3 Universidad de Magdalena-Colombia
}

Para citaciones: Del Río Cortina, A., \& Elías Caro, J. (2020). Dinámicas asociativas del tejido empresarial en Cartagena de Indias (1860-1960). Panorama Económico, 28(4), 215231. https://doi.org/10.32997/pe-2020-3326

Recibido: 2 de marzo de 2020

Aprobado: 19 de agosto de 2020

Autor de correspondencia: Abel Del Río Cortina adelrioc2311@universidadean.edu.co

Editor: Andrés Escobar E. Universidad de Cartagena-Colombia.

Tipología IBN Publindex: Artículo Resultado de Investigación

Copyright: (C) 2020. Del Río Cortina, A., \& Elías Caro, J. Este es un artículo de acceso abierto, distribuido bajo los términos de la licencia https://creativecommons.org/licenses/by-nc-

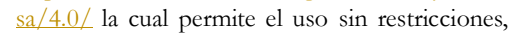
distribución y reproducción en cualquier medio, siempre y cuando que el original, el autor y la fuente sean acreditados.

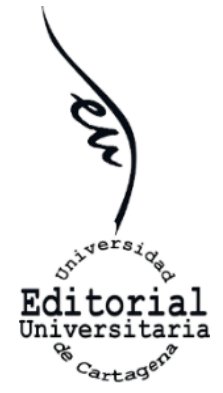

\section{RESUMEN}

En este documento, se considera a la familia Del Castillo como líder al interior de la red de empresarios conformada en Cartagena de Indias en el período de 1860-1960. La metodología utilizada para mostrar la red empresarial es análisis de redes sociales-ARS. Esta metodología, permitió evidenciar las dinámicas asociativas que originaron la construcción conjunta de flujos relacionales de recursos, bajo esquemas de amistad y de contacto familiar. Entre los resultados de la investigación, se muestra la importancia de la visualización de la conducta de los individuos a nivel micro, los patrones de relaciones enmarcados en la estructura de la red a nivel macro, y las interacciones entre los dos niveles, evidenciando, a su vez, las estructuras sociales que subyacen en los flujos relacionales, en los movimientos de recursos, y en las confluencias de poder, desde la perspectiva de las diferentes posiciones que surgen entre los actores de una red empresarial en contextos sociales específicos.

Palabras clave: Análisis de redes sociales; redes empresariales; dinámicas asociativas; historia empresarial.

JEL: M13, N86, N96.

\section{Associative dynamics of the business sector in Cartagena de Indias (1860-1960)}

\section{ABSTRACT}

In this paper we consider Del Castillo family as leader within the network of business entrepreneurs in Cartagena de Indias in the 1860-1960 period. The methodology used is the ARS-social network analysis to analyze the business network. This allow us to show the associative dynamics of resources, under economic relations and family contact schemes. The results suggests the importance of visualizing the behavior of individuals at the micro level, also the relationship patterns framed in the network structure at the macro level, and their interactions between these levels. We conclude that social structures underlie resource allocations and associative dynamics.

Keywords: Social network analysis; entrepreneurial networks; associative dynamics; specific social contexts; business history.

JEL: M13, N86, N96.

\footnotetext{
${ }^{1}$ El presente documento es parte de los resultados de la tesis de maestría titulada "Análisis del grupo familiar Del Castillo en relación al tejido empresarial de Cartagena de Indias (1860-1960)" (Del Río, 2012), como parte de los estudios en desarrollo empresarial impartidos por la Universidad del Magdalena.

2 PhD en Gerencia de Proyectos, Universidad EAN, Magister en Desarrollo Empresarial, Universidad del Magdalena. Email: adelrioc2311@universidadean.edu.co

3 Profesor de la Universidad del Magdalena, PhD en Ciencias Históricas, Universidad de la Habana. E-mail: jelias@unimagdalena.edu.co
} 


\section{INTRODUCCIÓN}

Existen evidencias de avances significativos en términos del desarrollo de documentos de historia empresarial en Colombia, los cuales, han permitido analizar temáticas como la historia empresarial y su incidencia económica, abordando a su vez, distintas dimensiones de los empresarios dentro de enfoques que combinan historia social, literatura de desarrollo económico, sociología, y elementos administrativos (Dávila, 2012), con la necesidad latente de profundizar en enfoques que permitan ampliar la comprensión de la estructura de pensamiento de los empresarios, en conjunto con la forma en que construyeron sus iniciativas, y en que dinamizaron el desarrollo del tejido productivo, considerando a autores como Sombart (1902), y Weber (1904), y planteamientos en cuanto al núcleo familiar propiciando el emprendimiento.

Se resalta la importancia de llegar a un balance en cuanto al análisis de la historia económica a partir de una visión macro, y de la perspectiva empresarial, para lo cual, se recomienda indagar en el mundo de los empresarios, considerando, de manera paralela la transformación de los negocios y de los diferentes sectores de la economía (Dávila, 2012).

Dávila (2012), deja reseñados los desafíos futuros acerca de la historiografía empresarial Latinoamericana y Colombiana, requiriéndose de la construcción de un vínculo más estrecho entre la investigación histórica, y los avances teóricos y analíticos de la comunidad internacional desde la business history, teniendo en cuanta la construcción de las empresas, las características fundamentales de los empresarios, e incluso, profundidad en los aspectos humanos de quienes se dedicaron a forjar iniciativas empresariales en contextos sociales particulares.

En este sentido, el análisis de redes sociales-ARS (Borgatti, Everett, \& Freeman, 2002), es una metodología desde la que es posible estudiar las conexiones configuradas como producto del contacto entre diversos actores, siendo estos, empresas, individuos, o territorios. Las conexiones se generan debido a que los actores participan de un proceso de intercambio de recursos, desarrollando actividades conjuntas, comunicación, colaboración, o procesos de competencia, lo cual, se ve reflejado en documentos o registros que permiten graficar una red de nodos que se expande o se contrae en el tiempo, emergiendo una red social, que, para el presente caso, sirve de apoyo al momento de analizar las diferentes interacciones de la familia Del Castillo como grupo empresarial líder en la red de empresarios de Cartagena de Indias (1860-1960).

Este documento se divide en cinco secciones, enfocándose en primera instancia, en el análisis de redes sociales como metodología de aproximación a la composición y evolución del tejido empresarial; luego, se presenta una revisión de diferentes autores concerniente a la historiografía en el Caribe Colombiano; se presentan las características de las dinámicas asociativas de la ciudad de Cartagena de Indias lideradas por la familia Del Castillo; se presenta el análisis de los resultados de estas dinámicas asociativas en el tiempo; $y$, finalmente, se muestran las conclusiones. 


\section{Iniciativas empresariales y Análisis de Redes sociales (ARS).}

El análisis de redes sociales-ARS (Borgatti, Everett, \& Freeman, 2002), es un soporte al momento de profundizar en los estudios de diferentes iniciativas empresariales gestadas en Cartagena de Indias en el período comprendido entre 1860 a 1960, considerando a la familia Del Castillo relevante en dicho desarrollo (Del Río, 2012).

El ARS es una metodología que conecta a los actores o participantes con la red social que se genera de las conexiones que los actores establecen. Con el fin de aplicar esta metodología, se tiene en cuenta que los marcos teóricos relevantes en ciencias sociales, muestran aproximaciones científicas de los fenómenos, ya sea, basados en modelos interpretativos de estructuras macro - sociales o económicas, o, considerando modelos intencionales explicativos de fundamentación micro, que tienen como centro las particularidades individuales de los participantes (Newman, Barabasi \& Watts, 2006).

Una red social se encuentra compuesta por nodos conformando conexiones sociales o nodales, las cuales, pueden ser interpersonales u organizacionales (Borgatti., et al, 2002). En este tipo de redes, se encuentran las redes del tejido empresarial, las cuales, son conformadas por una serie de actores productivos entre organizaciones, grupos familiares empresariales y empresarios, generando "conjuntos relacionales" a partir de conexiones de enfoque directo e indirecto. Las conexiones nodales se generan como producto de interacciones, intercambios, flujo de información, flujo de recursos, y procesos de comunicación, surgiendo dinámicas asociativas, que llevan a los participantes a desarrollar procesos de inclusión y de exclusión, los cuales, son determinados desde la estructura estratégica de cada actor participe, y, a su vez, son influenciados por las características de los diferentes sub-grupos que componen el tejido empresarial.

Los planteamientos de Veblen (1899) ilustran en cuanto a las motivaciones de los empresarios para desarrollar alianzas estratégicas, enmarcados en la "Teoría de la clase ociosa"; mientras que, los argumentos de Galtung (1964), muestran los condicionamientos que son aplicados por los empresarios desde la lógica social para desarrollar procesos de inclusión y exclusión, correspondiendo con dos de los axiomas planteados por el autor, los cuales son, 1. Congruencia del rango determinando un factor incremental de la asociatividad de los individuos; y, 2. Disposición asociativa de los individuos de rangos cercanos.

Las motivaciones empresariales, permiten adicionar las consideraciones de Sombart (1902) y Weber (1904), en cuanto a las bases del capitalismo, y de igual forma, los argumentos de Shumpeter (1942), en lo que corresponde a la creación destructiva como impulsor del cambio mediado por la figura del empresariado.

El análisis de redes cobra vigencia en la medida en que surgen enfoques sociológicos y antropológicos, que plantean la determinación del comportamiento de estructuras macrosociales, considerando las conexiones de individuos específicos, existiendo la necesidad de interpretación de las conexiones entre ambos niveles (Del Río, 2012). Lo anterior, se ve evidenciado, desde los años sesenta, en la utilización de grafos y formalismo matemático para representar las conexiones de redes, que, previamente se 
habían manejado mediante la identificación de relaciones intuitivas (Harary \& Norman, 1953).

El análisis de redes sociales -ARS, ha sido ampliamente utilizado en ciencias sociales, y en temas tan variados como epidemiología, evaluación psicosocial, comportamiento organizacional, y distribución de información (Borgatti., et al, 2002). De esta manera, las aplicaciones de mapeo organizacional, y de diferentes técnicas de mapeo cognitivo, han sido posibles debido a la evolución de las teorías de cognición enfocadas en las organizaciones (Eden \& Spender, 1998; Hodgkinson, 1997; Meindl, Stubbart \& Porac, 1996; Narayanan \& Kemmerer, 2001; Porac, Thomas \& Baden-Fuller, 1989), siendo extendidas hacia la mayor parte de temas de análisis en cuanto al desarrollo organizacional con la inclusión de aspectos relacionados con las tecnologías de la información (Nelson, Nadkarni, Narayanan \& Ghods, 2000).

Con el fin de generar el mapeo de las conexiones directas e indirectas, se utilizan diferentes programas computacionales de análisis de redes, entre los que tenemos Ucinet (Borgatti., et al, 2002), Pajeck (Batagelj \& Mrvar, 1996), y Siena (Ripley, Snijders, Boda, Vörös \& Preciado, 2013), entre otros.

Los resultados emergentes del análisis de redes permiten mejorar la comprensión de las acciones de los actores del tejido empresarial, con lo cual, es posible predecir las intervenciones de los actores, generando una mejor gestión del tejido como un ecosistema de implicaciones socio-económicas (Fujita, Krugman, \& Venables, 1999). Desde esta perspectiva, se consideran las conexiones entre los distintos actores productivos en redes compuestas por sub-grupos (Newman., et al, 2006), generando explicaciones de las relaciones entre actores, con un componente conductual micro a nivel de individuos, y un componente conductual macro desde el que se visualiza el comportamiento de la red, considerando de igual forma, la interacción entre el nivel micro y el macro.

\section{Revisión de literatura.}

Las condiciones históricas del tejido empresarial del Caribe Colombiano han sido reconstruidas por autores como Joaquín Viloria, Adolfo Meisel, Sergio Solano De Las Aguas, Jorge Elías Caro, y María Teresa Ripoll. Estos investigadores, han evidenciado la existencia de una red de empresarios que asumieron el desarrollo económico de un territorio con grandes potencialidades naturales, propicio para el comercio internacional y la industrialización.

Al momento de determinar la evolución económica del Caribe Colombiano, se aborda el texto: "Empresarios de Santa Marta: el caso de Joaquín y Manuel Julián de Mier, 1800 1896" (Viloria, 2000a), como un documento que presenta referencias valiosas con respecto a las ciudades de Santa Marta, Barranquilla y Cartagena, siendo un documento en el que se combinan elementos de estadística con apuntes de distintos investigadores relevantes, sirviendo de contexto para el análisis de las confrontaciones históricas entre estas tres ciudades. 
Se plantea la evolución del grupo empresarial familiar de Mier por un período de cien años (1800-1896), y, además, se aborda información descriptiva y de alto contenido estadístico.

Otro referente de evolución económica del Caribe Colombiano es el texto: "Banco de la República en Barranquilla, 1923-1951" (Viloria, 200b). Se detallan los aspectos de la creación y desarrollo del Banco de la República, enfocando la investigación en distintas sucursales del Caribe Colombiano, con énfasis en Barranquilla y Cartagena, mostrando aspectos políticos, sociales, y culturales.

El documento, aporta una comparación relevante de los movimientos de las sucursales de Barranquilla y Cartagena (1924-1929), evidenciando un proceso de letargo de la ciudad de Cartagena con respecto a Barranquilla.

En cuanto al desarrollo de ciertas ciudades desfavoreciendo el avance de otras, se presenta el escrito "Los Alemanes en el Caribe Colombiano: el caso de Adolfo Held, 1880 1927" (Meisel \& Viloria, 1999). Este texto, contiene elementos de gran importancia histórica en la medida en que muestra detalles de connotación política, en conjunto con aspectos sociales, culturales y económicos.

El documento, aporta detalles de singular importancia que permiten valorar la rivalidad histórica entre las ciudades de Santa Marta, Cartagena y Barranquilla, mostrando a su vez, detalles de la transformación en cuanto a las dinámicas asociativas empresariales en el Caribe Colombiano.

Finalmente, se aborda, como eje central del análisis del liderazgo del grupo familiar Del Castillo, el texto: "Empresarios centenaristas en Cartagena, cuatro estudios de caso", (Ripoll, 2007). Los fundamentos históricos de este documento, así como también, la información concerniente a cuatro iniciativas empresariales en las que participa la familia Del Castillo, lo hacen altamente importante para el desarrollo de la investigación.

El gran aporte del documento es la composición accionaria de las cuatro iniciativas, siendo estas, la Colombian Sugar Co (1906), la Cartagena Oil Refining Company (1909), la Compañía explotadora de petróleo (1913), y la Compañía de canalización del Dique (1919). La composición accionaria de estas iniciativas, permite reconstruir el tejido empresarial liderado por la familia Del Castillo con la aplicación del programa computacional UCINET, con lo cual, se genera la aproximación a las dinámicas asociativas del Caribe Colombiano en el período objeto de estudio.

De acuerdo a los argumentos de Veblen (1899), los grupos familiares empresariales del Caribe Colombiano desarrollaron un proceso de influencia político-económica, que toma como punto de partida la hazaña, con un alto componente militar, para luego, trascender hacia la industria, con una alta participación en diferentes sectores económicos, generando conexiones entre familias que se evidencian en la participación accionaria en empresas diversas de grupos familiares amigos, y que además, se observa en la asunción de riesgos compartidos y distribución de dividendos bajo parámetros de reinversión, y de ubicación de los excedentes de las operaciones en nuevas iniciativas de 
organizaciones empresariales de familias amigas, consolidando círculos cerrados de desarrollo empresarial.

Producto de lo anterior, se generan conexiones empresariales cerradas basadas en la confianza, y sobre todo, fundamentadas en la aceptación social. La riqueza, desde una perspectiva de opulencia desbordante, es mostrada de manera exquisita, suntuosa e inconmensurable, de esta forma, "...la riqueza, es sustentada como producto de calidades divinas, de estirpe, de abolengo..." (Del Río, 2012).

Las anteriores consideraciones, constituyen la base para mostrar las calidades individuales y familiares de generación de riqueza, llevando a una emulación con respecto a aquellos que poseen las condiciones para producir y mostrar la riqueza de forma ostensible (Veblen, 1899). De esta manera, se plantea una diferenciación de individuos y familias prominentes que muestran sus bienes con un consumo ostensible.

En este sentido, se muestra la manera en que diferentes familias del Caribe Colombiano asumen un lujo desbordante y exquisito, lo cual, le apunta al desarrollo de una imagen social que se encuentra impulsando la adquisición de propiedades diversas a nivel nacional e internacional, los constantes viajes al exterior, la educación de los diferentes miembros de la familia en el exterior con énfasis en Estados Unidos e Inglaterra, las reuniones de gala y los banquetes, mostrando una solidez financiera que permite la atracción de un mayor número de inversionistas, y la generación de un mayor respeto social (Del Río, 2012).

En la narración planteada por Ripoll (2007), se muestra la manera en que diferentes grupos familiares prominentes ubicaron a sus hijos en colegios y universidades en Estados Unidos, generando a su vez, movilidad familiar hacia Europa, con énfasis en la identificación de posibles socios, y en el mejoramiento de las condiciones de generación de riqueza como estrategia empresarial.

Las conexiones, basadas en la confianza, se acentúan con los flujos comerciales permanentes desarrollados en el Caribe Colombiano entre 1860 y 1960. Este auge del comercio resulta en la consolidación de grupos familiares empresariales con iniciativas conjuntas.

El conjunto relacional de individuos u organizaciones representados por los grupos familiares empresariales, y por las iniciativas en que invirtieron, se representa en una red social en la que se evidencian las conexiones directas e indirectas, que muestran el surgimiento de dinámicas asociativas, en las cuales, se presentan dinámicas incluyentes y excluyentes, permitiendo la identificación de estructuras relacionales con propiedades sistémicas (Del Río, 2012). Las estructuras que emergen de la red social permiten la revisión de información que mejora la comprensión de las interacciones humanas y organizacionales (Newman., et al, 2006).

Los grupos familiares empresariales del período posterior a la independencia presentan una continuidad de las prácticas coloniales, esto, debido a una construcción de nación basada en un proyecto de la élite, que incide en la manera de concebir y desarrollar 
política (Melo, 1992), lo cual, configura un escenario propicio para las iniciativas empresariales de las familias prominentes.

De acuerdo con los planteamientos de Galtun (1964), la sociedad puede ser estructurada mediante la asunción de roles específicos, teniendo cada role, un rango distintivo, y permitiendo la aparición de dinámicas incluyentes y excluyentes. En cuanto a estas dinámicas, se presentan procesos de cohesión, que, según el autor, se estructuran en dos clases sociales, la baja y la alta. Se plantean una serie de axiomas regulatorios de las relaciones, resaltándose dos de estos axiomas, como son, la igualdad de rangos como base para los procesos de conexión y asociación; y, la preferencia de rangos similares por desarrollar procesos de asociación (Galtun, 1964).

El equilibrio social, con miras a la construcción de sociedades equitativas y productivas, requiere de la implementación de un tercer axioma, el cual, se basa en el desarrollo de actores conciliadores que establezcan puentes de contacto entre los actores interrelacionados. Los actores conciliadores, generan dinámicas de inclusión y exclusión, que, desde un dinamismo propio del sistema socioeconómico permiten la revitalización del tejido, tanto social como empresarial, sin aplicar acciones violentas (Galtun, 1964).

Los empresarios del Caribe Colombiano en el período objeto de estudio, desarrollaron una serie de iniciativas bajo esquemas de relaciones de confianza e interdependencia, combinando aspectos políticos, económicos, y socioculturales, con lo cual, impactan en el territorio y adquirieren posiciones respetables ${ }^{4}$ (Del Río, 2012).

La élite capitalista cartagenera, conformó estructuras relacionales, interconectando grupos familiares empresariales que participaron en diferentes iniciativas conjuntas. Lo anterior, se evidencia en los aportes de los autores que sirven de base a la presente investigación.

En cuanto al tercer axioma, la élite cartagenera desarrolló procesos de intervención en política mediante posturas obrero-patronales que favorecieron la evolución de las empresas, pero, a su vez, fortalecieron el desarrollo social dentro de posturas liberales, interviniendo como mediadores en procesos de violencia generados a nivel regional y nacional, en diferentes períodos (Del Río, 2012).

\section{Dinámicas asociativas en Cartagena de Indias.}

En Cartagena de Indias se generaron una serie de dinámicas asociativas en el período de 1860 a 1960, que, en primera instancia generaron un tejido empresarial de impacto regional, y que luego, trascendieron a nivel nacional con conexiones internacionales (Del Río, 2012). Estas dinámicas y sus conexiones pueden ser graficadas aplicando análisis de redes sociales-ARS (Borgatti., et al, 2002), como metodología desde la que es posible ubicar actores estratégicos de la red empresarial.

Lo anterior, permite generar estudios más exhaustivos a partir del liderazgo del grupo familiar Del Castillo, que desarrolló inversiones en las cuatro iniciativas enunciadas

\footnotetext{
4 De acuerdo con Del Río (2012), el análisis del proceso de conformación de la nación es de vital importancia para llegar
} a comprender el escenario que rodeo a los empresarios del Caribe Colombiano entre los años de 1860 a 1960. 
anteriormente, con un 17,65\% de participación en la Cartagena Oil Refining Company, compañía gestada por su amigo, Diego Martínez Camargo (Ripoll, 2007), siendo su segundo accionista, de acuerdo con la información del periódico "El Porvenir" de mayo 5 de 1909, seguida esta posición accionaria, de la correspondiente a la Compañía de Canalización del Dique con un 5,08\% en 1919, para luego ubicar, la Compañía Explotadora de Petróleos de Cartagena con un 2,30\% de acuerdo a su acta de creación en 1913, y finalmente, la Colombian Sugar Co con un porcentaje accionario de 0,009\%, de acuerdo a la información contenida en el texto: "El licenciado Serpentín", (1955) Fig 1.

Figura 1. Empresas Interrelacionadas en la ciudad de Cartagena (1860-1960)

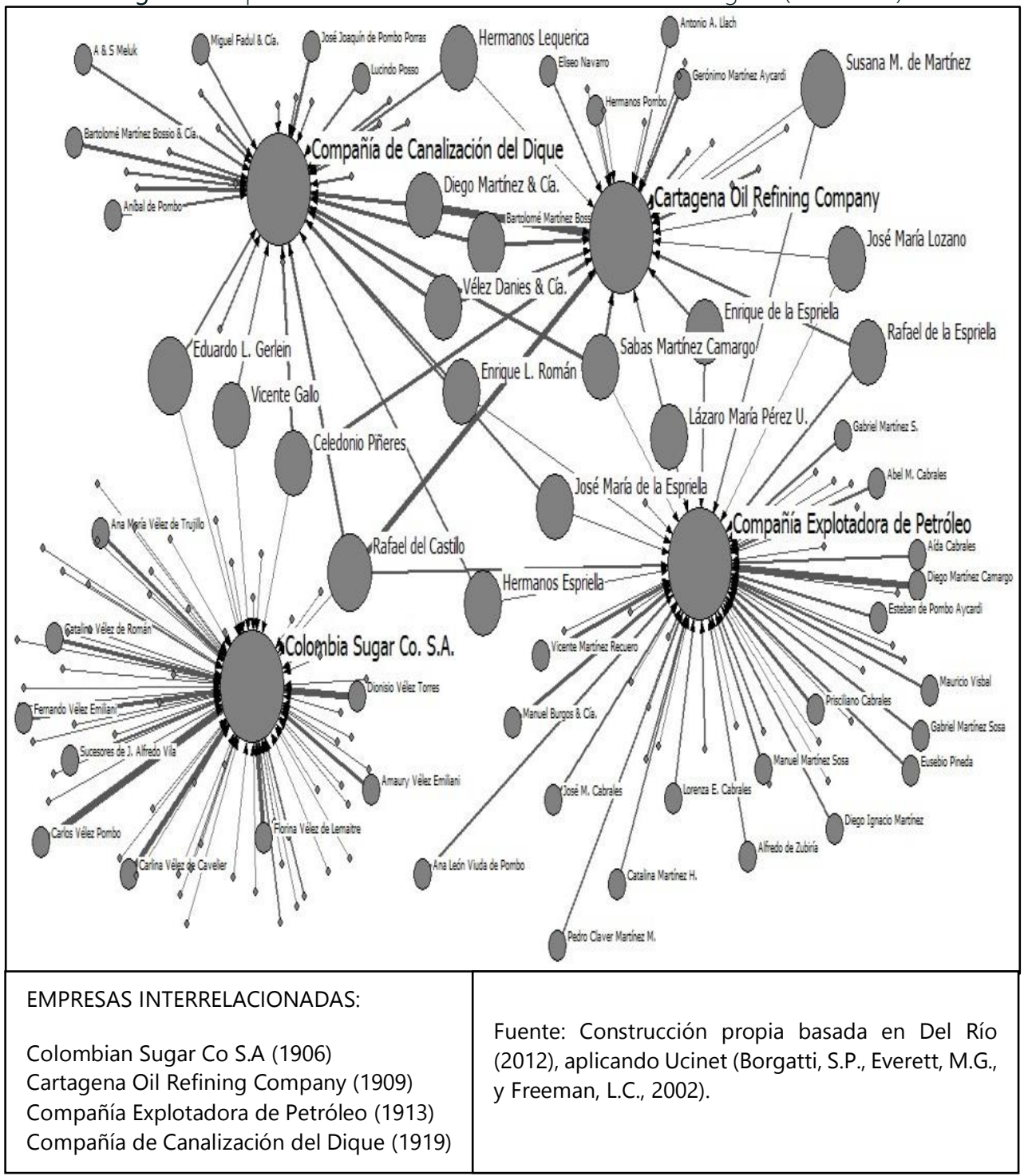

${ }^{5}$ La Figura 1, es una representación de cuatro iniciativas empresariales de gran importancia gestadas en el Caribe Colombiano, siendo estas, la Colombian Sugar Co, S.A. (1906), la Cartagena Oil Refining Company (1909), la Compañía Explotadora de Petróleo (1913), y, finalmente, la Compañía de Canalización del Dique (1919). En la construcción de la figura, se tomó la información de los protocolos notariales con la composición accionaria correspondiente a las organizaciones en cuestión, evidenciándose diferencias en los aportes de los participantes en las iniciativas, esto, a partir del grosor de los vértices correspondientes, siendo los nodos los diferentes accionistas. El marco de referencia es el Texto: Empresarios Centenaristas en Cartagena, (Ripoll, 2007). 
Los aportes estadísticos planteados en el análisis se derivan de la aplicación del software Ucinet (Del Río, 2012), de forma tal, que se muestran los cuatro nodos que se conforman con la participación accionaria de los grupos familiares en las iniciativas empresariales.

En estos datos estadísticos, se evidencia que la iniciativa empresarial que representa una menor conexión al interior de la red es la Colombian Sugar Co S.A o ingenio azucarero Central Colombia, con un grado de medida central de 7; mientras que la Cartagena Oil Refining Company, y la Compañía de canalización del dique, presentan un grado de medida central de 17, y la Compañía Explotadora de petróleo, presenta un grado de 15, Fig. 2.

Figura 2. Matriz de estadística descriptiva y de medidas centrales de Freeman. ${ }^{6}$

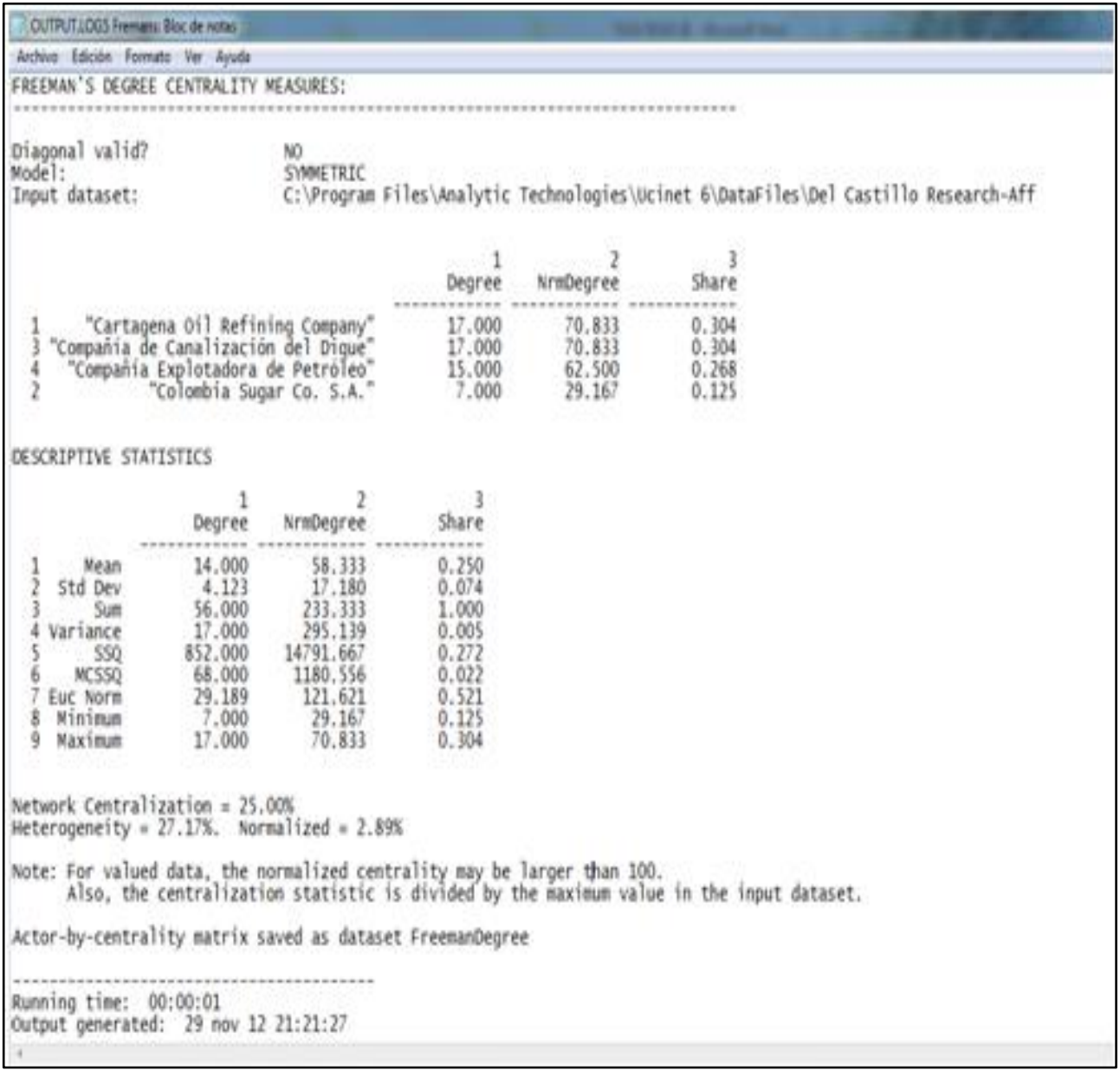

Fuente: Del Río (2012), aplicando Ucinet (Borgatti, Everett, \& Freeman, 2002).

De esta forma, se observa el grado de interconexión de los diferentes grupos familiares en términos de centralidad, Fig 3. Así como también, es posible identificar el grupo empresarial que presenta menor número de actores por fuera de la familia que lidera la iniciativa, siendo, la Colombian Sugar Co S.A., representando una alta concentración de poder en manos del grupo familiar Vélez, Fig. 4.

${ }^{6}$ La Figura 2, representa los elementos estadísticos y de medidas centrales que permite identificar el grado de interrelación de las diferentes iniciativas. 
Figura 3. Grupos familiares empresariales interconectados.

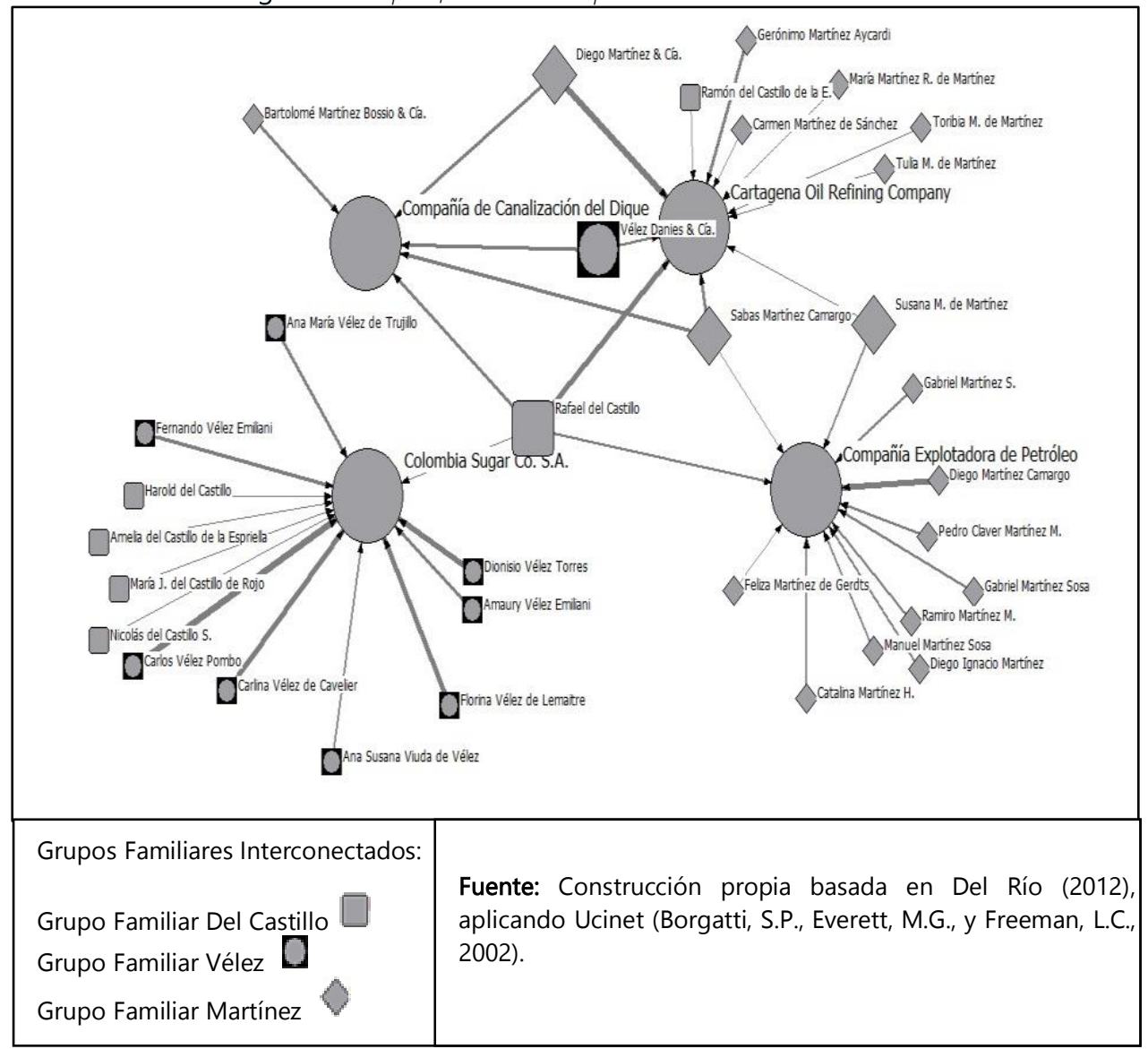

Figura 4. Colombian Sugar Co S.A.

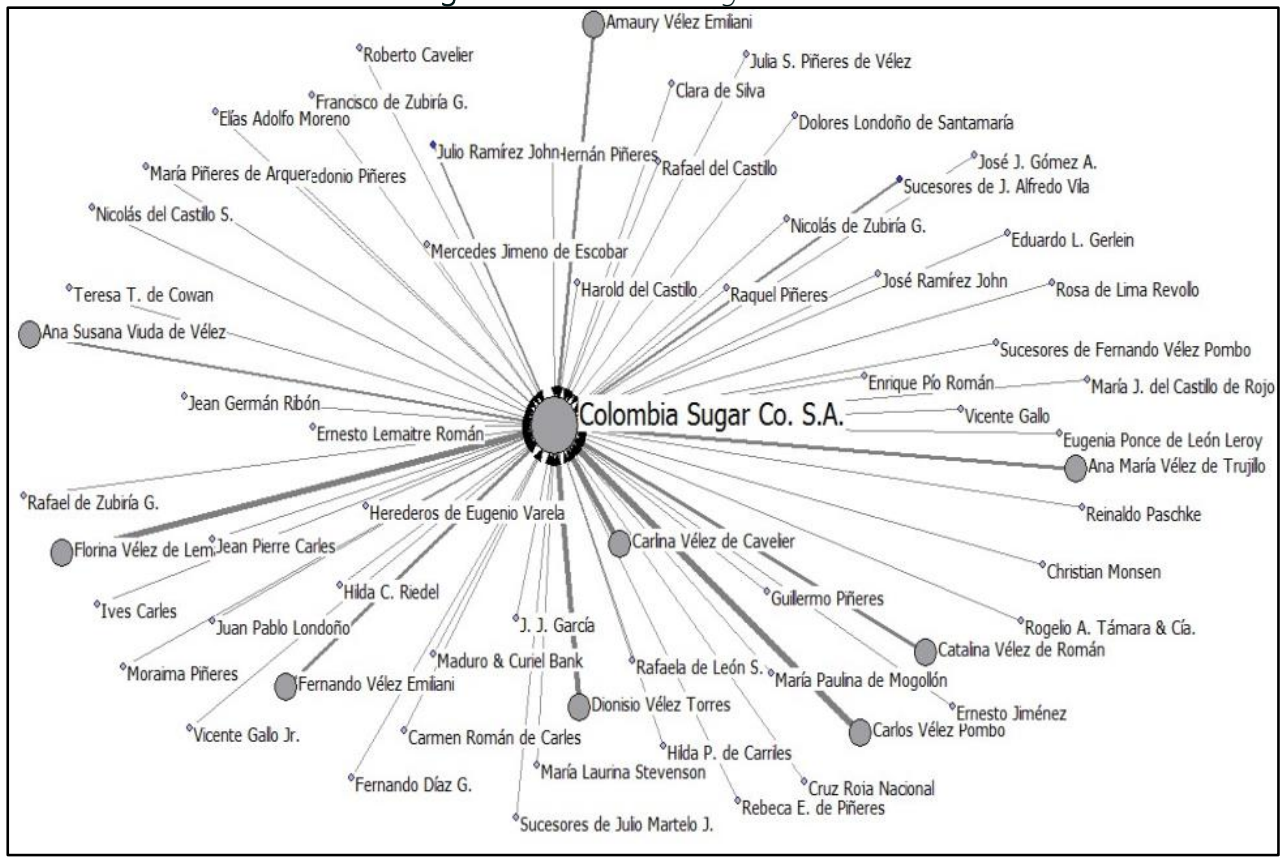

Fuente: Construcción propia basada en Del Río (2012), aplicando Ucinet (Borgatti, Everett, \& Freeman, 2002). 
Se evidencia la importancia del grupo familiar Del Castillo, en la medida en que manejó operaciones en diferentes territorios, tanto nacionales como internacionales. En el año 1913 se destacan las locaciones de Nueva York, en coordinación con oficinas en Cartagena, con una serie de sucursales y conexiones en Medellín, Bogotá, Ibagué, Manizales, Cúcuta, Cali, Barranquilla y Santa Marta, en conjunto con los mercados regionales del Sinú, Sabanas de Bolívar y márgenes del río Atrato (Del Río, 2012).

Las conexiones internacionales de la familia Del Castillo se extienden, ubicándose en Centroamérica y el Caribe en diversos destinos, tales como, Panamá, Cuba, San Salvador, Santo Domingo, Haití, Puerto Rico y las Antillas Británicas, manejando, a su vez, sucursales y socios en Venezuela, Ecuador y Filipinas (Ripoll, 2007, p.146-147).

El grupo familiar empresarial Del Castillo posee una posición estratégica en el tejido empresarial del período objeto de estudio (Del Río, 2012). Este grupo familiar, desarrolla inversiones en diferentes iniciativas empresariales amigas a nivel local, regional, nacional e internacional, con énfasis en el desarrollo regional.

Desde el origen de la casa comercial Del Castillo en 1861, bajo la dirección de Rafael Del Castillo Del Castillo, se evidencia la tenacidad de la familia. Las condiciones económicas de Cartagena al momento del inicio de la casa comercial eran de pobreza e incertidumbre; sin embargo, los negocios progresaron de manera rápida (Ripoll, 2007).

Es posible observar la capacidad de riesgo de los grupos familiares del Caribe Colombiano, en la forma como incursionan en diversas iniciativas de con miras a participar del mercado aledaño a la ciudad de Cartagena, llegando a las principales regiones de Bolívar grande, y a los principales territorios nacionales, en conjunto con locaciones internacionales, capacidad de asunción de riesgos que se muestra en la evolución de la familia Del Castillo (Tabla 1).

Tabla 1. Momentos de evolución del Grupo familiar empresarial Del Castillo (1861-1960).

\begin{tabular}{|c|c|c|c|c|}
\hline $\mathrm{N}^{\circ}$ & Momento & Razón Social & Responsable & Observaciones \\
\hline 1 & $1861-1890$ & $\begin{array}{l}\text { Rafael Del Castillo } \\
\text { \& Cía }\end{array}$ & $\begin{array}{l}\text { Rafael Del Castillo } \\
\text { Del Castillo }\end{array}$ & $\begin{array}{l}\text { Inicio de la casa comercial y apertura de } \\
\text { mercados. }\end{array}$ \\
\hline 2 & $\begin{array}{l}1891- \\
1902\end{array}$ & $\begin{array}{l}\text { Rafael Del Castillo } \\
\text { \& Cía. }\end{array}$ & $\begin{array}{l}\text { Rafael C. y Carlos } \\
\text { Del Castillo De La } \\
\text { Espriella }\end{array}$ & $\begin{array}{l}\text { Reorganización del negocio para el cambio } \\
\text { generacional. }\end{array}$ \\
\hline 3 & 1903 & $\begin{array}{l}\text { Rafael Del Castillo } \\
\text { \& Cía. }\end{array}$ & $\begin{array}{l}\text { Rafael C. y Carlos } \\
\text { Del Castillo De La } \\
\text { Espriella }\end{array}$ & $\begin{array}{l}\text { Proceso de inestabilidad en el papel } \\
\text { moneda- Ley } 33 \text { de } 1903 .\end{array}$ \\
\hline 4 & 1908 & $\begin{array}{l}\text { Rafael Del Castillo } \\
\text { \& Cía. }\end{array}$ & $\begin{array}{l}\text { Rafael Del Castillo } \\
\text { De La Espriella }\end{array}$ & $\begin{array}{l}\text { Fallecimiento de Rafael Del Castillo Del } \\
\text { Castillo y conformación de una nueva } \\
\text { sociedad. }\end{array}$ \\
\hline 5 & 1913 & $\begin{array}{l}\text { Rafael Del Castillo } \\
\text { \& Cía. }\end{array}$ & $\begin{array}{l}\text { Rafael Del Castillo } \\
\text { De La Espriella }\end{array}$ & $\begin{array}{l}\text { Desarrollo de cuenta conjunta entre las } \\
\text { operaciones de Nueva York y Cartagena. }\end{array}$ \\
\hline 6 & 1917 & $\begin{array}{l}\text { Rafael Del Castillo } \\
\text { \& Cía. }\end{array}$ & $\begin{array}{l}\text { Rafael Del Castillo } \\
\text { De La Espriella }\end{array}$ & $\begin{array}{l}\text { Reorganización debido a los flujos de } \\
\text { recursos derivados de las operaciones } \\
\text { desarrolladas en Nueva York. }\end{array}$ \\
\hline 7 & 1925 & $\begin{array}{l}\text { Rafael Del Castillo } \\
\text { \& Corporation. }\end{array}$ & $\begin{array}{l}\text { Rafael Del Castillo } \\
\text { De La Espriella }\end{array}$ & $\begin{array}{l}\text { Conformación de una nueva compañía } \\
\text { debido a los flujos desarrollados desde } \\
\text { Nueva York. }\end{array}$ \\
\hline 8 & 1939 & $\begin{array}{l}\text { Rafael Del Castillo } \\
\text { \& Corporation. }\end{array}$ & $\begin{array}{l}\text { Ramón Del Castillo } \\
\text { De La Espriella }\end{array}$ & $\begin{array}{l}\text { Reorganización bajo el liderazgo de las } \\
\text { familias Del Castillo Crawford, y Del Castillo }\end{array}$ \\
\hline
\end{tabular}




\begin{tabular}{|c|l|l|l|l|}
\hline$N^{\circ}$ & Momento & Razón Social & \multicolumn{1}{|c|}{ Responsable } & \multicolumn{1}{c|}{ Observaciones } \\
\hline & & & & $\begin{array}{l}\text { De La Espriella, después de la muerte de } \\
\text { Carlos y Rafael Del Castillo De La Espriella. }\end{array}$ \\
\hline 9 & $1940-1945$ & $\begin{array}{l}\text { Rafael Del Castillo } \\
\text { \& Corporation. }\end{array}$ & $\begin{array}{l}\text { Ramón Del Castillo } \\
\text { De La Espriella }\end{array}$ & $\begin{array}{l}\text { Reorganización a partir de la incursión en la } \\
\text { industria harinera. }\end{array}$ \\
\hline 10 & $1948-1960$ & $\begin{array}{l}\text { Rafael Del Castillo } \\
\text { \& Corporation. }\end{array}$ & $\begin{array}{l}\text { Rafael Del Castillo } \\
\text { Stevenson. }\end{array}$ & $\begin{array}{l}\text { Reorganización a partir del fallecimiento de } \\
\text { Ramón Del Castillo De La Espriella. }\end{array}$ \\
\hline
\end{tabular}

Fuente: Del Río (2012)

La magnitud de la participación del grupo familiar empresarial en la evolución del tejido productivo de la ciudad de Cartagena, se visualiza en la diversidad de los negocios que desarrolló, en las ubicaciones geográficas en que tuvo operaciones, y en las conexiones que generó.

En cuanto a su incidencia político-militar, el grupo familiar fue protagonista de diversos acontecimientos históricos apoyando a las instancias gubernamentales. Durante la guerra de los mil días, la familia Del Castillo desarrolló una participación relevante, sirviendo en la mediación para la adquisición de armamento prestando recursos financieros y facilitando sus navíos con la intervención de la Compañía W.D. Munson, obteniendo como contraprestación los derechos de importación de diversos bienes (Ripoll, 2007).

En el mejoramiento del canal del Dique (1919), el grupo Del Castillo participó, en conjunto con las familias más prestigiosas de Cartagena, como son, Velez Daníes, Jaspe, Pombo, Martínez Camargo, Piñeres, Mainero, Meluk, Dáger, Covo, Román, y Espriella, entre otras. El foco de sus negocios internacionales fue la ciudad de Nueva York, siendo, esta ciudad, el punto de conexión que sirvió de enlace de consolidación de sus importaciones y exportaciones. En Nueva york, se apoyó la iniciativa de actividad petrolera dirigida por Don Diego Martínez Camargo, quien fuera socio y amigo de Rafael Del Castillo de la Espriella. El grupo familiar empresarial Del Castillo se encargó de la identificación de la maquinaria que se requería para la explotación de petróleo, generando los contactos de importación para la primera refinería de origen Colombiano (Ripoll, 2007).

Las familias empresariales Cartageneras del período analizado se apartaron de la ocupación de cargos públicos, sin embargo, muchas de sus acciones giraron alrededor del poder político, apoyando, particularmente, en los períodos presidenciales de Rafael Reyes (1904-1909), Carlos E. Restrepo (1910-1914), y Pedro Nel Ospina (1922-1926). Los grupos familiares Cartageneros mantuvieron relaciones de amistad y de negocios, que, en su momento, incidieron en el auge de la región caribe durante estos períodos presidenciales, con apoyo gubernamental (Ripoll, 2007).

La evolución del grupo familiar Del Castillo, y, en general, de los grupos familiares empresariales de la ciudad de Cartagena del período de estudio, muestra la configuración de una mentalidad diferente, la cual, concibe el desarrollo desde la participación en la industrialización y en la intervención con una óptica de modernización.

En este nuevo momento histórico, el urbanismo comienza a tener una alta importancia, con los retos que esto implica, con lo cual, la modernización industrial incide en el

\footnotetext{
${ }^{7}$ La presente tabla es extraída de la tesis de maestría titulada: "Análisis del grupo familiar Del Castillo en relación al tejido empresarial de Cartagena de Indias (1860-1960)" (Del Río, 2012).
} 
desarrollo urbano con la fábrica impulsando el desarrollo económico, dirigido por el entonces presidente Rafael Núñez.

"...En el proceso del segundo período presidencial de Rafael Núñez (1884-1886), se genera, debido a diferencias ideológicas, uno de los conflictos más devastadores para el país como es la guerra de los mil días. Al finalizar la guerra, se busca consolidar un conjunto de reformas, bajo el proceso de regeneración. Este es un momento de soporte político que incide en la transformación empresarial del país, con un esquema de manejo centralizado del poder que persiste hasta nuestros días..."(Del Río, 2012).

La industrialización se presenta como un proceso truncado, esto, debido a divergencias permanentes que generaron divisiones, y causaron dificultades para la coexistencia de los diferentes territorios, dificultando, a su vez, la cohesión nacional requerida para el fortalecimiento del tejido productivo.

Las divisiones se originaron debido al legado de la independencia, en cuanto a líderes regionalistas que buscaron prolongar los privilegios que se habían consolidado en la época colonial. Estas divisiones requieren ser analizadas abordando las condiciones políticas de desarrollo de la recién creada república (Del Río, 2012).

Ripoll (2007), muestra la relación entre los grupos familiares empresariales y el poder político, con una significativa participación en el mejoramiento de las condiciones del Caribe Colombiano, lo que se manifiesta en la consigna de "Menos política y más administración" manifestada por Reyes en 1904.

\section{Análisis y Discusión}

El esquema de visualización longitudinal desarrollado mediante análisis de redes socialesARS, permite mostrar la relevancia del liderazgo de la familia Del Castillo en la estructuración del tejido productivo de Cartagena de 1860 a 1960.

La familia Del Castillo, participó en diversos negocios iniciando con la comercialización de productos importados en 1861, los cuales, distribuyó a nivel local, regional y nacional, para luego, incursionar en iniciativas de industrialización en 1873, desarrollando exportación de materias primas en 1878, ofreciendo servicios bancarios de comercialización de oro en 1878, finca raíz en 1887, inversiones en infraestructura con un astillero en 1891, inversiones en transporte fluvial en 1897, representación de casas comerciales extranjeras en 1900, y, proporcionando apoyo a diversos grupos familiares en 1900 .

La familia Del Castillo desarrolló inversiones en la Cartagena Oil Refining Company y en el Ingenio Central Colombia en 1908, liderados por la familia Vélez Daníes, y de igual manera, en Extractos Tánicos en 1911, con conexiones comerciales hacia la zona central del país con la participación de grupos empresariales familiares del centro del país (19051935).

Desde 1908, Nueva York fue el centro de conexión con los territorios internacionales, generando transacciones bursátiles, y, estableciendo operaciones en Sur América, Centro 
América, y el Caribe en 1912, invirtiendo en la explotación de petróleo en 1913, en fabricación de hielo, en producción de cerveza y en la canalización del Dique en 1919, importando arroz desde Siam (Tailandia) en 1926, siendo negociador de la deuda con Estados Unidos en 1931, invirtiendo en bonos del gobierno nacional y en acciones del Banco Hipotecario de Colombia en 1939, y, finalmente, participando de la industria harinera con un molino que se encuentra funcionando la actualidad (Del Río, 2012).

Existen evidencias, en cuanto a que el desarrollo económico del Caribe Colombiano en el período de 1860 a 1960 fue interrumpido por diferentes aspectos, como son, una marcada dependencia de exportaciones carentes de valor agregado, importaciones de productos suntuarios, con pocos elementos de transformación productiva, alto endeudamiento regional, poca planeación con respecto a vías de acceso, servicios públicos, y educación, políticas gubernamentales centralizadas, y desarrollo precario de la industria, que, con la excepción de Barranquilla, presentaba un desarrollo incipiente.

La ciudad de Barranquilla, como excepción, presentó una transformación con alto movimiento comercial desde 1870. La anterior característica, atrajo a diversas familias de Cartagena, Santa Marta y del extranjero, presentando una consolidación de la actividad industrial a inicios del siglo XX (Del Río, 2012), con La fábrica de tejidos Obregón en 1909, Cervecería de Barranquilla en 1913, Banco Dugand en 1917, empresa de aviación Scadta en 1919, y Compañía urbanizadora El Prado en 1920 (Viloria, 2000b).

En cuanto a la ciudad de Cartagena, el período de 1860 a 1960 fue de gran auge, resaltándose el proceso de diversificación gestionado por los ganaderos de Bolívar con características de adopción de riesgos y liderazgo. En este período, Cartagena se visualizaba como un territorio de industrialización, con grupos familiares empresariales, que, desde una visión estratégica, invirtieron en fábricas, industrias y organizaciones empresariales reconocidas, que con el cambio generacional fueron perdiendo la consolidación competitiva.

\section{Conclusiones}

Al analizar el tejido empresarial, la identificación de actores influyentes en su construcción y desarrollo es importante; sin embargo, desde la concepción del análisis de redes sociales ARS, se requiere, de forma adicional, del análisis de las estructuras en las que se encuentran los actores estratégicos, siendo estas, conformadas por un entramado de red relacional. La comprensión de las acciones de los actores influyentes al interior de una red con conexiones diversas, permite la generación de estrategias de fomento de condiciones que sirvan de base para el fortalecimiento del sector productivo, centrando los esfuerzos en el estudio de los lazos al interior de la red, con la consecuente generación de oportunidades significativas y restricciones de inclusión. La manera en que se encuentre estructurada la red va a permitir su evolución, en términos de oportunidades o de restricciones significativas.

Las condiciones del Caribe Colombiano a finales del siglo XIX, y comienzos del siglo XX, son de empobrecimiento al interior del período republicano, representando para Cartagena la pérdida de su posición como puerto principal. En ese momento histórico, la ciudad atraviesa circunstancias desfavorables producto de la guerra, la baja navegabilidad 
del canal del Dique, y el descenso demográfico. Las importaciones, en conjunto con el transporte marítimo y fluvial, son considerados como medios de reactivación económica acogidos Rafael Núñez, a finales del siglo XIX, consiguiendo auge desde el siglo XX, con representantes de importancia, como son: Rafael del Castillo, Diego Martínez Camargo, Los hermanos Vélez Danies, Juan B. Mainero y Trucco, los hermanos Pombo, Bartolomé Martínez Bossio, y Nicolas de Zubiría, entre otros.

Las conexiones empresariales entre los diferentes grupos familiares, fueron fortalecidas mediante relaciones de amistad que perduran hasta nuestros días, posibilitando, en su momento, las bases del tejido productivo. Además de lo anterior, la posición geográfica del Caribe Colombiano con ciudades tan emblemáticas, como Cartagena, Barranquilla y Santa Marta, permitió la confluencia de una gran diversidad cultural y de intercambio de ideas de distintos lugares a nivel mundial producto del contacto comercial, que, paulatinamente dio origen a una industrialización inicial de impacto local, regional y nacional. El Caribe Colombiano, en el período de la república, continuó siendo la puerta de entrada del flujo comercial con los distintos intercambios culturales y de negocio que esto representaba.

Los grupos familiares empresariales del Caribe Colombiano desarrollaron una serie de conexiones que implicaron alianzas organizacionales compartiendo responsabilidades y riesgos, que permitieron una mejor distribución de utilidades. Bajo estas alianzas se generaron inversiones en diferentes iniciativas de familias amigas, en la misma vía de lo propuesto por Thorstein Veblen (1899), autor que plantea que los empresarios tienen el poder de generar transformación enfocada en cambios sociales. Este poder ha sido ejercido tomando como base el poder militar, para luego, llegar al poder político y económico.

De otra parte, Shumpeter (1942), plantea la creación destructiva como el ejercicio de transformación gestionado por los empresarios, lo cual, genera dinámicas de inclusión y exclusión, que, desde el tejido empresarial analizado, se evidencian en los lazos de amistad presentes entre los accionistas de las diferentes iniciativas empresariales. La teoría de Galtun (1964), aborda los fundamentos de los procesos de inclusión y exclusión, de tal forma, que se consideran una serie de reglas desde las que es posible analizar el comportamiento de los actores de la red empresarial.

En este sentido, la familia Del Castillo participó activamente en el desarrollo de la red de empresarios de Cartagena en el período de 1860 a 1960, con un enfoque de alianzas estratégicas con grupos familiares empresariales con los que sostuvo relaciones de amistad (Ripoll, 2007). Lo anterior, es la base para la generación de una red empresarial soportada en confianza mutua y el liderazgo familiar.

La reconstrucción de los escenarios empresariales en el período entre 1860 y 1960, es un punto de inicio para visualizar la historia empresarial de Cartagena, considerando los vínculos entre los diferentes actores que hicieron posible el auge de la ciudad, y, de igual manera, es un análisis que permite visualizar algunas de las razones del estancamiento posterior a dicho período. 
Finalmente, el análisis del empresariado es un aspecto relevante para llegar a la comprensión de la realidad económica y social, teniendo como centro a los actores que produjeron el cambio respaldado por la capacidad de riesgo, y por la confianza que generaban.

\section{Referencias}

Batagelj V., Mrvar A. (1998). Pajek. A Program for Large Network Analysis. Connections, 21 (2), 47 57. https://doi.org/10.1007/978-3-642-18638-7

Borgatti, S.P., Everett, M.G., and Freeman, L.C. (2002). UCINET 6 for Windows: Software for social network analysis. Harvard: Analytic Technologies. Recuperado de: https://sites.google.com/site/ucinetsoftware/home

Dávila De Guevara, C. (2012). Empresariado en Colombia: perspectiva histórica y regional, Bogotá, Universidad de los Andes, Ediciones Uniandes. Recuperado de: http://hdl.handle.net/1992/46609

Del Río, A. A. (2012). Análisis del grupo familiar Del Castillo en relación al tejido empresarial de Cartagena de Indias (1860-1960), Tesis de grado de Magister en Desarrollo empresarial. Universidad del Magdalena. Recuperado de: http://repositorio.unimagdalena.edu.co/jspui/handle/123456789/4107

Eden, C., \& Spender, J. C. (1998). Managerial and organizational cognition. Sage, London.

Fujita, M., Krugman, P. y Venables, A.J. (1999): The spatial economy: Cities. regions and international trade. MIT Press, Cambridge MA.

Galtung, J. (1964). Rango e Integración social: una aproximación multidimensional, Anales de la Facultad de Ciencias Sociales (FLACSO), 1(1).

Harary, F., Norman, R. Z. (1953). Graph Theory as a Mathematical Model in Social Science. Ann Arbor, Mich: Institute for Social Science. Recuperado de: http://www.idiosophy.com/wpcontent/uploads/2017/07/harary-norman.pdf

Hodgkinson, G. P. (1997). Cognitive inertia in a turbulent market: The case of UK residential estate agents. Journal of Management Studies, 34, 921-945. https://doi.org/10.1111/1467-6486.00078

Licenciado S. (1955). Los secretos del inspector Caraballo. Historia mínima, sintética, anecdótica, pintoresca, cómica, y trágica de la vida, pasión y muerte de la Colombian Sugar Company. Bogotá: Iqueima.

Meindl, J.R., Stubbart., C., Porac, J. F. (1996). Cognition within and between organisations, London: Sage.

Meisel, A. (1999). Los alemanes en el Caribe Colombiano: el caso de Adolfo Held, 1880 1927, Cuadernos de Historia Económica 005364, Banco de la República. Recuperado de: https://www.banrep.gov.co/es/alemanes-el-caribe-colombiano-el-caso-adolfo-held-1880-1927

Narayanan, V. K., Kemmerer, B. (2001). A cognitive perspective on strategic management: Contributions, challenges, and implications. Academy of Management Conference 2003, Washington, DC.

Nelson, K.M., Nadkarni, S., Narayanan, V.K., Ghods, M. (2000). Understanding software operations support expertise: A revealed causal mapping approach. MIS Quarterly, 24, 475-507. Recuperado de: https://misq.org/understanding-software-operations-support-expertise-arevealed-causal-mapping-approach.html 
Newman, M., Barabasi, A. L., Watts, D. J. (2006). The Structure and Dynamics of Networks. Princeton University Press.

Porac, J. F., Thomas, H., Baden-Fuller, C. (1989). Competitive groups as cognitive communities: The case of Scottish knitwear manufacturers. Journal of Management Studies, 26, 397-416. https://doi.org/10.1111/j.1467-6486.1989.tb00736.x

Ripley, R., Snijders, T.A.B., Boda, Z., Vörös, A., Preciado, P. (2013). Manual for RSiena (version 1.1250). http://www.stats.ox.ac.uk/ snijders/siena/RSiena_Manual.pdf

Ripoll, M. T. (2007). Empresarios centenaristas en Cartagena, Cuatro estudios de caso. Cartagena: Ediciones Unitecnológica.

Schumpeter, Joseph A. (1942). Capitalism, Socialism and Democracy. London: Unwin.

Sombart, W. (1946). El Apogeo del Capitalismo. México: Fondo de Cultura Económica.

Veblen, T. (1973). Theory of the leisure class. Boston, MA: Houghton Mifflin Co.

Viloria, J. (2000a). Empresarios de Santa Marta: el caso de Joaquín y Manuel Julián de Mier, 18001896, Cuadernos de Historia Económica 002577, Banco de la República. Recuperado de: https://www.banrep.gov.co/es/empresarios-santa-marta-el-caso-joaquin-y-manuel-julianmier-1800-1896

Viloria, J. (2000b). Banco de la República en Barranquilla, 1923-1951, Cuadernos de Historia Económica 002047, Banco de la República. Recuperado de: https://www.banrep.gov.co/es/banco-republica-barranquilla-1923-1951

Weber, M. (1969). La Ética Protestante y el Espíritu del Capitalismo. Barcelona: Ediciones Península. 